\title{
Book review: Grandfathers
}

\author{
HANS HÄMÄLÄINEN, University of Helsinki, Finland
}

Buchanan, A \& Rotkirch, A. (Eds.) (2016).

Grandfathers: Global Perspectives. London: Palgrave MacMillan.

In the last decades, grandparents and their role in family life have been widely studied in the different fields of family studies. However, in family research, grandfathers have received much less attention than grandmothers. The book in question makes a refreshing exception and focuses on grandfathers. According to the editors Ann Buchanan and Anna Rotkirch, the aim of the book is to bring grandfathers out of the shadows of grandmothers and make them visible by providing a comprehensive account of grandfather studies. To reach this goal, the editors gathered an international group of scholars from different disciplines to explore grandfatherhood from various perspectives.

The book consists of 16 chapters divided into five sections. The first part is an overview, which contains an introduction and interdisciplinary context on grandfatherhood, incorporating research from evolutionary biology, sociology, psychology, and economics. The second part explores cultural circumstances influencing grandfathering by presenting a historical investigation and case studies from different countries and cultures (China, Denmark, New Zealand, the UK, and the US). The third part of the book explores the factors associated with involved grandfathering by utilizing international (11 European countries) and national datasets (Australia and Finland). Part four includes only one chapter that examines prior studies on the effects of grandchild involvement on grandfathers in North America. The fifth section concentrates on the grandfathers' impact on the well-being of their grandchildren using data from England, Israel, South Africa, and Wales. In the last chapter of the book, the editors summarize the findings of the chapters and discuss new insights as well as outline some policy implications based on the presented results.

A notable merit of the book is that it provides multidisciplinary and cross-cultural perspectives on grandfathers. The independent studies featured in the book utilize multiple different approaches and methods, and therefore the book provides a comprehensive viewpoint on grandfatherhood. This book can be recommended for researchers, teachers, and students in all fields of research who are concerned with intergenerational family relations. In addition, because the book is written in a clearly worded manner, it is suitable reading for anyone who is interested in grandfathers or grandparenthood in general. 
As always, some shortcomings can be found in the book. Despite the title of the book, 'Global Perspectives', most of the chapters are focused on Western or high-income countries, and only a few chapters explore grandfathers in other regions or cultures. Another limitation is related to methodological issues. All the empirical studies analyse either cross-sectional or qualitative datasets, and none of them utilize longitudinal statistical data. As the editors (e.g., Chapter 16) and many of the authors of individual chapters note, studies based on cross-sectional data do not provide information about the causal relationships of events, such as grandfather involvement and grandchildren well-being. Longitudinal data and methods would also be needed to investigate the changes over time, within family generations, and across individual life courses. For example, cross-sectional data from different years show that there are changes in the roles of grandfathers, although longitudinal methods are required to analyse the change and the factors behind it as well as individual variations.

However, by no means do the abovementioned shortcomings indicate a failure of the book. The book reaches its aims to provide a comprehensive account of current grandfather studies, and it truly pulls grandfathers out from the shadows for further investigations. The strong merits of the book were also noticed by the British Academy, which nominated the volume for the Peter Townsend Prize 2017. The abovementioned limitations are due to the lack of prior grandfather studies and the scarcity of available empirical data on grandfathers, especially outside high-income countries. Hence, this book can be perceived as a pioneering work, which hopefully promotes further and more detailed research on grandfatherhood. 